\title{
Evolution of the Fermi surface of a doped topological insulator with carrier concentration
}

\author{
E. Lahoud, ${ }^{1}$ E. Maniv, ${ }^{2}$ M. Shaviv Petrushevsky, ${ }^{2}$ M. Naamneh, ${ }^{1}$ A. Ribak, ${ }^{1}$ S. Wiedmann, ${ }^{3}$ L. Petaccia, ${ }^{4}$ Z. Salman, ${ }^{5}$ \\ K. B. Chashka, ${ }^{1}$ Y. Dagan, ${ }^{2, *}$ and A. Kanigel ${ }^{1}$ \\ ${ }^{1}$ Physics Department, Technion-Israel Institute of Technology, Haifa 32000, Israel \\ ${ }^{2}$ Raymond and Beverly Sackler School of Physics and Astronomy, Tel-Aviv University, Tel Aviv 69978, Israel \\ ${ }^{3}$ High Field Magnet Laboratory, Institute for Molecules and Materials, Radboud University Nijmegen, Toernooiveld 7 , \\ NL-6525 ED Nijmegen, The Netherlands \\ ${ }^{4}$ Elettra Sincrotrone Trieste, Strada Statale $14 \mathrm{~km}$ 163.5, 34149 Trieste, Italy \\ ${ }^{5}$ Laboratory for Muon Spin Spectroscopy, Paul Scherrer Institut, CH-5232 Villigen PSI, Switzerland \\ (Received 30 July 2013; revised manuscript received 14 October 2013; published 5 November 2013)
}

\begin{abstract}
In an ideal bulk topological insulator (TI) conducting surface states protected by time-reversal symmetry enfold an insulating crystal. However, the archetypical $\mathrm{TI}, \mathrm{Bi}_{2} \mathrm{Se}_{3}$, is actually never insulating; it is in fact a relatively good metal. Nevertheless, it is the most studied system among all the TIs, mainly due to its simple band structure and large spin-orbit gap. Recently, it was shown that copper intercalated $\mathrm{Bi}_{2} \mathrm{Se}_{3}$ becomes superconducting and it was suggested as a realization of a topological superconductor. Here we use a combination of techniques that are sensitive to the shape of the Fermi surface (FS): the Shubnikov-de Haas effect and angle-resolved photoemission spectroscopy to study the evolution of the FS shape with carrier concentration, $n$. We find that as $n$ increases, the FS becomes two-dimensional-like. These results are of crucial importance for understanding the superconducting properties of $\mathrm{Cu}_{x} \mathrm{Bi}_{2} \mathrm{Se}_{3}$.
\end{abstract}

DOI: 10.1103/PhysRevB.88.195107

PACS number(s): 71.18.+y, 71.20.-b, 79.60.-i

\section{INTRODUCTION}

A topological superconductor is a state of matter in which the bulk is fully gapped, but gapless surface states host Bogoliubov quasiparticles. ${ }^{1,2}$ Point-contact experiments have shown the existence of zero bias conductance peaks (ZBCP) in $\mathrm{Cu}_{x} \mathrm{Bi}_{2} \mathrm{Se}_{3},{ }^{3,4}$ and these were interpreted as a signature of Andreev surface bound states that were theoretically predicated to exist in certain classes of topological superconductor (TSCs). ${ }^{5,6}$ While the topological properties of topological insulator (TIs) are set by the band structure and should not depend on the chemical potential, ${ }^{7}$ the properties of the superconducting samples are sensitive to the chemical potential and to the shape of the Fermi surface (FS). ${ }^{5}$

$\mathrm{Bi}_{2} \mathrm{Se}_{3}$ has carriers in the conduction band even when carefully prepared. ${ }^{8}$ These carriers are believed to be the result of $\mathrm{Se}$ vacancies which are always present in the material. ${ }^{9}$ The carrier concentration can be increased further by $\mathrm{Cu}$ intercalation. ${ }^{10,11}$ The band structure of $\mathrm{Bi}_{2} \mathrm{Se}_{3}$ is three dimensional (3D), i.e., there is substantial electronic dispersion in the $k_{z}$ direction. On the other hand, the material is layered, cleaves easily, and its resistivity is anisotropic with $\rho_{z z} / \rho_{x x} \simeq 10 .{ }^{12}$ Band structure calculations $(\mathrm{BSC})^{7}$ indicate that the $\Gamma Z$ dispersion is weaker than the $\Gamma L$ dispersion. Early angle-resolved photoemission spectroscopy (ARPES) experiments have shown that the dispersion can be even weaker than the BSC predictions. ${ }^{13}$ It therefore is plausible that, on adding charge carriers, the FS will grow in an anisotropic fashion, where $k_{F}$ along the $k_{z}$ direction becomes considerably larger than $k_{F}$ in the $k_{x} k_{y}$ plane. The FS therefore can change from being a closed spherical FS at low carrier densities into an open cylinder-like $\mathrm{FS}$ at high carrier densities. An important question is whether $\mathrm{Cu}_{x} \mathrm{Bi}_{2} \mathrm{Se}_{3}$ has a closed or open FS at carrier densities in which the system is superconducting, $n \simeq 10^{20} \mathrm{~cm}^{-3}$.
The Shubnikov-de Haas ( $\mathrm{SdH}$ ) effect probes extrema in the cross section of the FS. According to the Onsager relation, the frequency of the magnetoresistance oscillation as a function of inverse magnetic field is $F=\frac{\hbar}{2 \pi e} A\left(\epsilon_{F}\right),{ }^{14}$ with $A\left(\epsilon_{F}\right)$ being the maximal cross-sectional area of the FS in a plane perpendicular to the magnetic field. By rotating the field with respect to the crystal, one can map the full momentum dependence of the FS.

Another common method for studying the FS is ARPES, which was found to be an ideal tool for studying the topological insulators. ARPES allows one to observe directly the surface states even in samples where the transport is dominated by the bulk..$^{15}$ On the other hand, the surface of a topological-insulator is a very complicated environment for photoemission; the ARPES spectrum consists of contributions from the bulk bands and surface states and possibly from a confined $2 \mathrm{D}$ electron gas. ${ }^{16}$ One way to disentangle these contributions is to scan the photon energy used in the experiment. Different photon-energies provide information about the dispersion at different $k_{z}$ values and allow one to distinguish 2D-like bands from the 3D bulk band.

In this work we use the two powerful experimental tools, SdH and ARPES; their combination allows us to determine the evolution of the $\mathrm{FS}$ as a function of carrier concentration. Using ARPES we show that the Dirac surface states exist throughout the carrier concentration range under study. However, only parts of the bulk-band FS can be clearly seen using ARPES due to the photon-energy dependence of the matrix elements. The SdH effect, in principle, allows a direct mapping of the entire FS but its amplitude depends exponentially on the effective mass. Consequently, it is less sensitive to high-bandmass regions on the FS. Despite the limitations of both probes, together they bring deep insight into the shape and properties of the FS. 

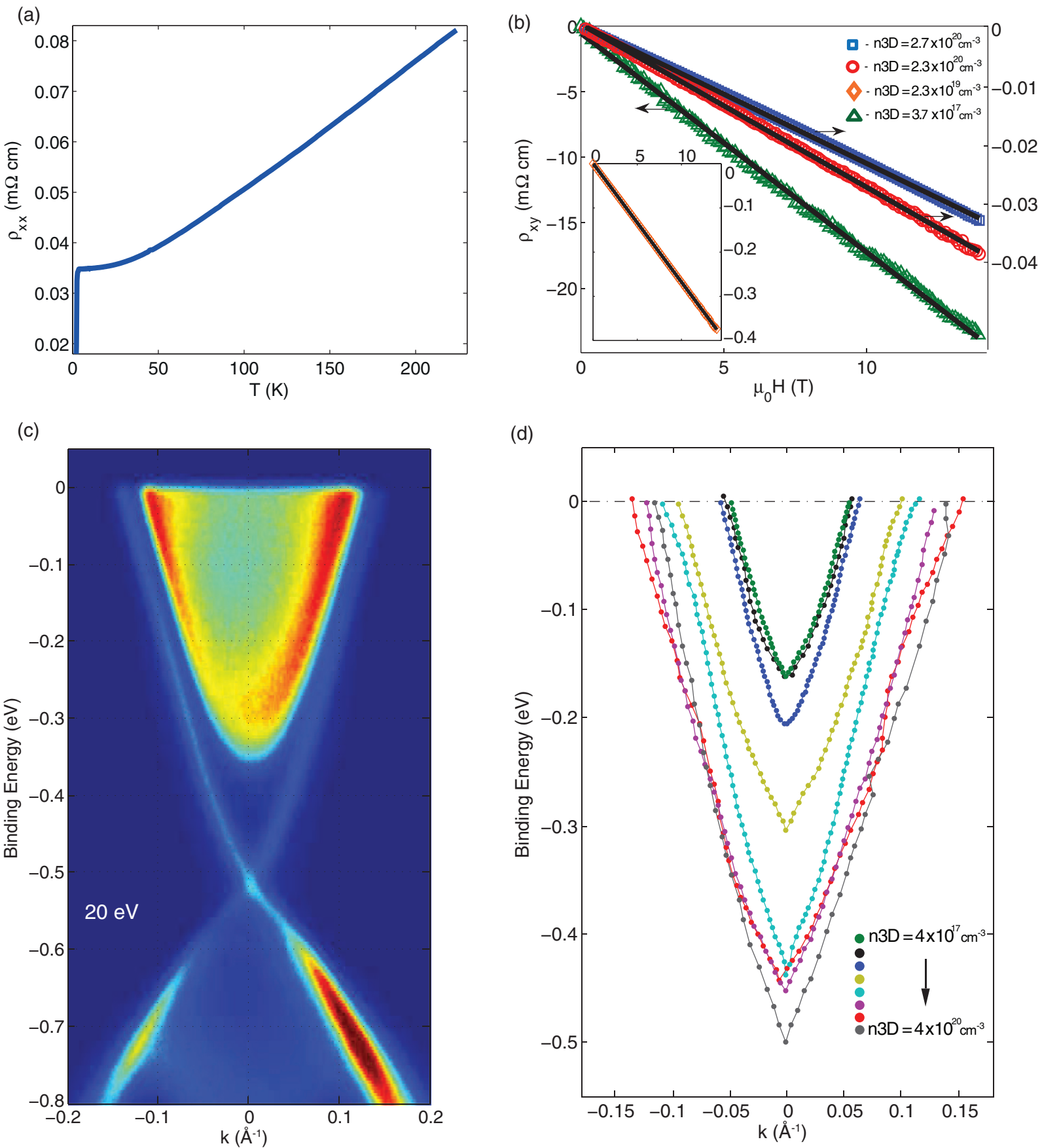

FIG. 1. (Color online) Transport and ARPES characterization. (a) Longitudinal resistivity versus temperature for $\mathrm{Cu}$-doped $\mathrm{Bi}_{2} \mathrm{Se}_{3}$ with $n \simeq 10^{20} \mathrm{~cm}^{-3}$. Metallic type behavior as well as supercoductivity below $\simeq 3 \mathrm{~K}$ is observed. Different samples may exhibit various superconducting volume fractions and $T_{c}$ variations. (b) Transverse resistivity versus magnetic field at $2 \mathrm{~K}$ for three samples. The solid lines are linear fits from which we extract the carrier concentration $n$. Inset: The same for $n \simeq 10^{19} \mathrm{~cm}^{-3}$. (c) Typical ARPES data from a highly doped sample measured with $20-\mathrm{eV}$ photon energy. The detector image shows the dispersion along the $\Gamma-\mathrm{K}$ direction. One can see that both the surface-state and the bulk-band dispersion coexist. (d) Surface-state dispersion as measured using ARPES for eight different samples with carrier concentrations ranging from $4 \times 10^{17} \mathrm{~cm}^{-3}$ (green curve) to $4 \times 10^{20} \mathrm{~cm}^{-3}$ (gray curve).

\section{METHODS}

For this experiment, we prepared a series of $\mathrm{Bi}_{2} \mathrm{Se}_{3}$ samples with different carrier concentrations. We used two kind of samples: off-stoichiometric $\mathrm{Bi}_{2-x} \mathrm{Se}_{3+y}$ with low carrier density $\left(n \simeq 10^{17}-10^{19} \mathrm{~cm}^{-3}\right)$, and $\mathrm{Cu}$ intercalated $\mathrm{Bi}_{2} \mathrm{Se}_{3}$ with high carrier density ( $n \simeq 10^{20} \mathrm{~cm}^{-3}$ ). All the samples were prepared using the modified Bridgeman method as described in Refs. 4 and 17. For transport measurements, flakes $\sim 1-30 \mu \mathrm{m}$ thick were freshly cleaved perpendicular to the $\mathrm{C} 3$ axis in a nitrogen environment. Gold contact wires were attached to the samples using silver paint. Hall measurements up to 

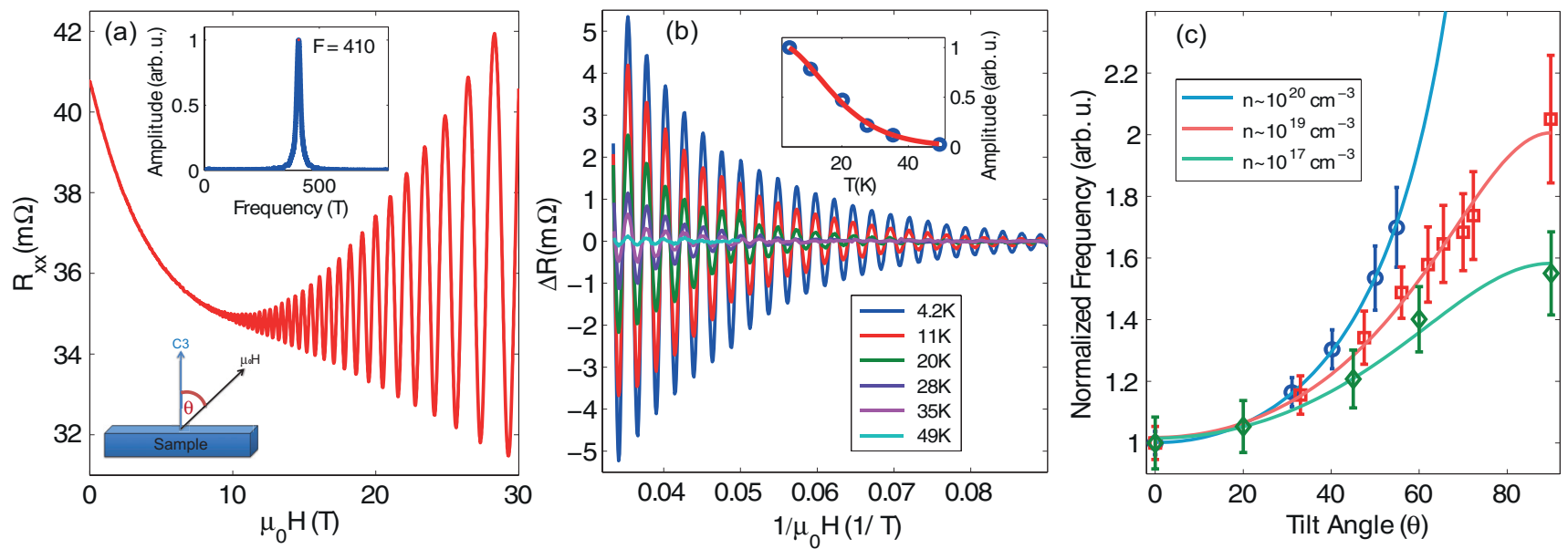

FIG. 2. (Color online) SdH data and analysis. (a) Longitudinal resistance versus magnetic field applied parallel to the C3 axis $(\theta=0)$ at $4.2 \mathrm{~K}$ for $n \simeq 10^{20} \mathrm{~cm}^{-3}$ (sample A). Inset: Fast Fourier transform (FFT) of these data plotted versus $\frac{1}{\mu_{0} H}$ after subtracting a smooth polynomial background. The sharpness of the FFT peak indicates a well-defined frequency. Its full width at half maximum is used as an upper limit for the uncertainty in determining the frequency. A drawing of the sample configuration used in this experiment is also shown. (b) Resistance versus $\frac{1}{\mu_{0} H}$ after subtraction of a smooth polynomial background at various temperatures [4.2 $\mathrm{K}$ data are taken from Fig. 2(a)]. The field is applied parallel to the $\mathrm{C} 3$ axis. Inset: Effective mass is extracted by following the oscillation amplitude at high field as a function of the temperature. The solid line is a fit to the Dingle formula, ${ }^{14}$ yielding $\mathrm{m} * \simeq 0.24 m_{e}$ for this sample $\left(n \simeq 10^{20} \mathrm{~cm}^{-3}\right)$. (c) The frequency as determined from the FFT versus tilt angle $\theta$ between the magnetic field and the $\mathrm{C} 3$ axis for three carrier concentrations. Solid lines are fits for an ellipsoidal FS $\left(n \simeq 10^{17}, 10^{19} \mathrm{~cm}^{-3}\right)$ and for a cylindrical FS $\left[F \propto \frac{1}{\cos (\theta)}\right]$ for $n \simeq 10^{20} \mathrm{~cm}^{-3}$ (sample B).

$14 \mathrm{~T}$ were performed using dc technique. $\mathrm{SdH}$ measurements up to $30 \mathrm{~T}$ were performed at the HFML using the standard lock-in technique. The thickness of the flakes was measured in a scanning electron microscope. The ARPES data were measured at the PGM beamline at the Synchrotron Radiation Center (SRC) (Stoughton, WI) and at the BaDEIPh beamline at Elettra (Trieste Italy). All the samples were cleaved at base temperature $(\sim 20 \mathrm{~K})$ in a vacuum better than $5 \times 10^{-11}$ torr and measured at the same temperature. Each sample was measured for no more than $6 \mathrm{~h}$; within this time we did not observe any change in the chemical potential.

\section{RESULTS AND DISCUSSION}

Typical resistivity versus temperature and low temperature Hall measurements are shown in Figs. 1(a) and 1(b), respectively. Typical ARPES data from a $\mathrm{Cu}$ intercalated sample with $n \simeq 4 \times 10^{20} \mathrm{~cm}^{-3}$ are shown in Fig. 1(c). A well-defined surface state can be seen, with a Dirac point at about $500 \mathrm{meV}$ below the Fermi level. The two linearly dispersive surface-state branches enclose the parabolic bulk band whose FS is the subject of this paper. Figure 1(d) shows the dispersion of the surface states for various samples with different carrier concentrations $n$. Upon changing $n$, the Dirac (a)

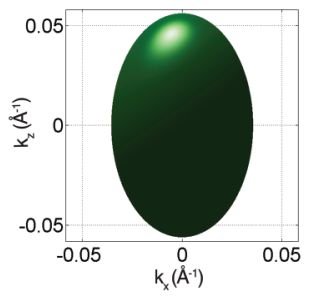

(e)

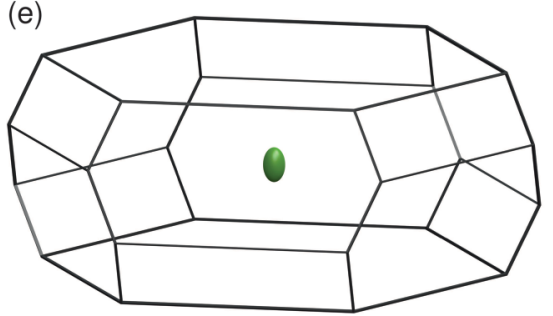

(b)

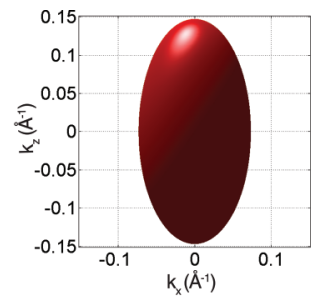

(c)

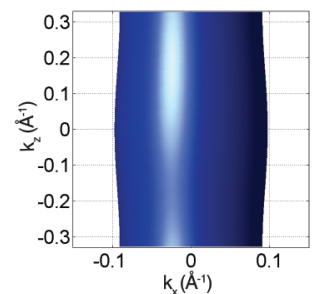

(f)

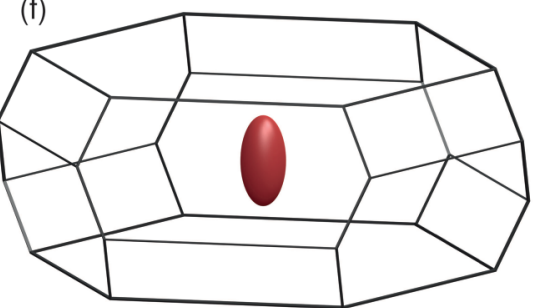

(d)

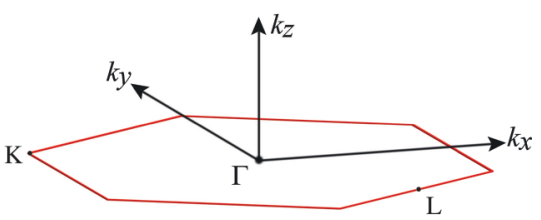

(g)

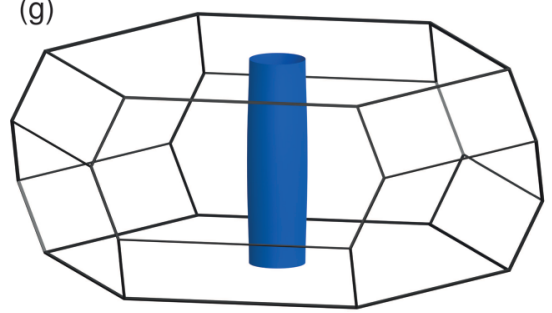

FIG. 3. (Color online) Evolution of the Fermi surface with carrier concentration. [(a) and (b)] Calculated ellipsoidal FS from the SdH data in Fig. 2(c), for $n \simeq 10^{17}, 10^{19} \mathrm{~cm}^{-3}$, respectively. Detailed profile view of the Fermi surfaces is shown. (c) Calculated FS using tight binding corrugated cylinder model fit to the $\mathrm{SdH}$ data in Fig. 2(c) (sample $\mathrm{B}, n \simeq 10^{20} \mathrm{~cm}^{-3}$ ) (see Appendix E for more information). (D) The Brillouin-zone momenta axes. [(e)-(g)] The Fermi surfaces of (a)-(c), respectively, plotted to scale with respect to the Brillouin zone. 

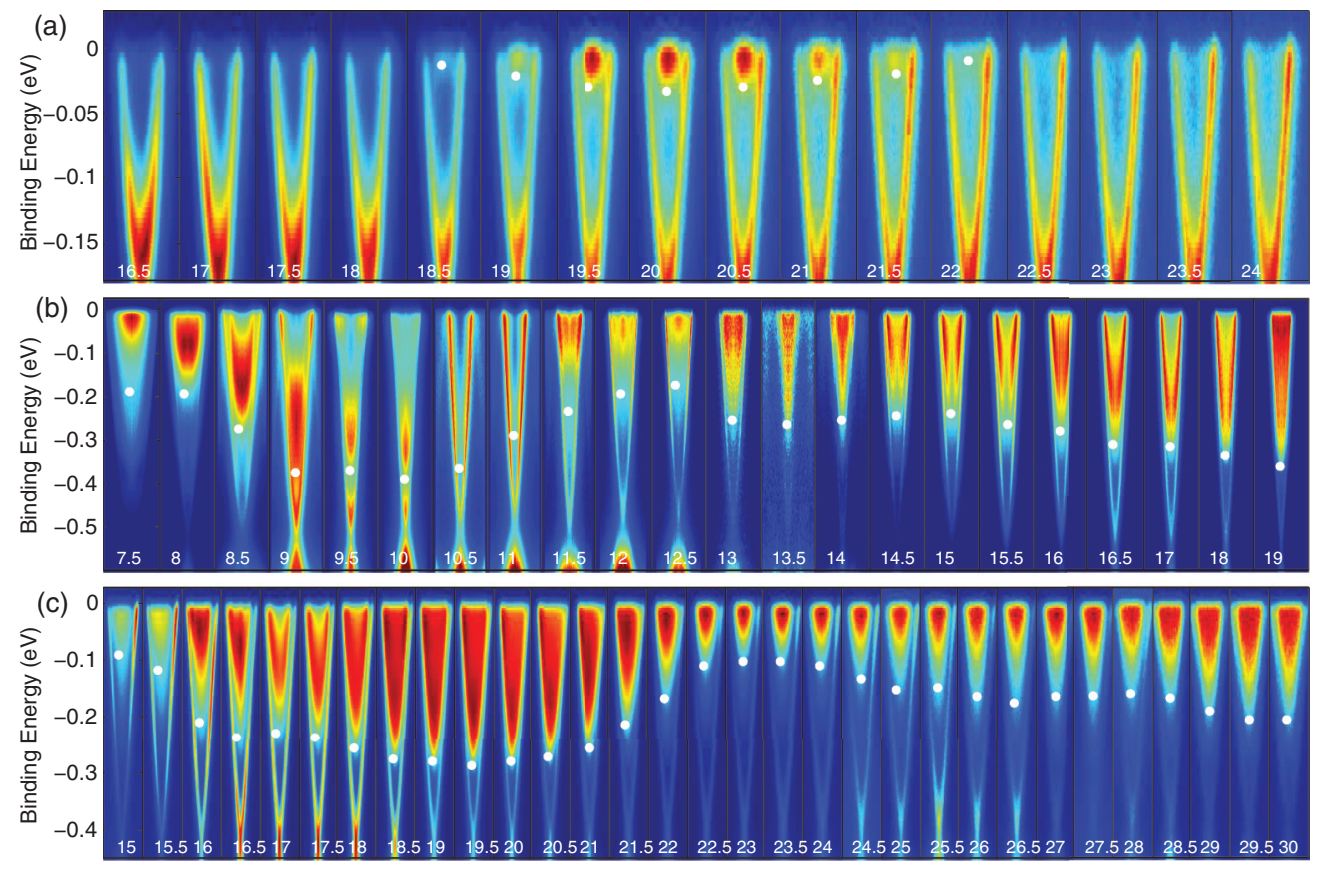

FIG. 4. (Color online) Photon energy dependence of the ARPES data. We show normal emission data for three different samples: (a) $n=4 \times 10^{17} \mathrm{~cm}^{-3}$, (b) $n=4 \times 10^{20} \mathrm{~cm}^{-3}$, and (c) $n=2 \times 10^{20} \mathrm{~cm}^{-3}$. The white dots represent the bottom of the bulk band. For the low-carrier-density sample the bulk band is seen only around $19 \mathrm{eV}(\Gamma$ point $)$, and for the the high-carrier-density samples the bulk band is visible at the entire photon-energy range measured. In panel (b) we show low-photon-energy data, believed to be more bulk sensitive. We find that the bulk band is visible at the entire photon-energy range measured, which covers a momentum range larger than the $\Gamma-\mathrm{Z}$ separation.

dispersion remains intact with a rigid shift of the Dirac point towards lower energies, while the Fermi velocity (i.e., the slope) remains unchanged.

In Fig. 2 we show $\mathrm{SdH}$ data [see lower inset of Fig. 2(a) for the configuration used in this experiment]. The resistance as a function of magnetic field for a highly doped sample ( $n \simeq 10^{20} \mathrm{~cm}^{-3}$ ) is shown in Fig. 2(a). Clear SdH oscillations can be seen. The Fourier transform of these oscillations is shown in the upper inset. A single, well-defined frequency is observed. The oscillations persist up to surprisingly high temperatures [see Fig. 2(b)]. From the temperature dependence of the amplitude, the effective mass $m^{*} \simeq 0.24 m_{e}$ is extracted [see the inset in Fig. 2(b)]. In Fig. 2(c) we show the angular dependence of the $\mathrm{SdH}$ frequency for three samples. For the low-carrier-concentration samples the oscillations persist up to a tilt angle of $90^{\circ}$ [see Fig. 2(c)], indicative of a closed ellipsoidal FS. These results are in agreement with previous observations. ${ }^{15,18-20}$ The FS of the $n \simeq 10^{19} \mathrm{~cm}^{-3}$ is clearly more elongated than the FS of $n \simeq 10^{17} \mathrm{~cm}^{-3}$. For $n \simeq 10^{20} \mathrm{~cm}^{-3}$ the oscillation amplitude decreases with increasing angle and cannot be observed beyond an angle of $55^{\circ}$. This angular dependence of the $\mathrm{SdH}$ frequency follows almost perfectly $F \propto \frac{1}{\cos (\theta)}$, which is the dependence expected for a cylindrical FS. ${ }^{22}$

In Fig. 3 we show the FS of these samples as reconstructed using the $\mathrm{SdH}$ data from Fig. 2(c). The first two samples with $n \simeq 10^{17}, 10^{19} \mathrm{~cm}^{-3}$, have an ellipsoidal FS. For the third sample ( $n \simeq 10^{20} \mathrm{~cm}^{-3}$ ) we fit our data to a simplified corrugated-cylinder model (see Appendix E for more information). The resulting FS is shown in Figs. 3(c) and 3(g). Our $\mathrm{SdH}$ data suggest a transition in the shape of the FS from a closed ellipsoid to an open FS as $n$ increases. Below we show that the ARPES data verify this effect.

In an ARPES experiment the signal intensity allows a direct mapping of the electronic dispersion along momentum directions which are parallel to the sample surface. This is because only the in-plane momentum is conserved. To map the dispersion along $k_{Z}$, one needs to scan the photon energy. We used the free-electron final-state approximation ${ }^{21}$ to find the correspondence between the photon energy and $k_{Z}$ (see Appendix B for more information).

In order to map the dispersion along the $k_{z}$ direction, we performed ARPES measurements at normal emission over a wide range of photon energies in steps of $0.5 \mathrm{eV}$. This was done for various samples with different carrier concentrations. A set of scans is shown in Fig. 4(a) for a $n \simeq 10^{17} \mathrm{~cm}^{-3}$ sample and in Fig. 4(b) and 4(c) for two highly doped samples, $n \simeq 10^{20} \mathrm{~cm}^{-3}$. One can see in Fig. 4 that, as expected, the 2D surface states are insensitive to the photon energy used.

For the low- $n$ sample shown, the bulk band is visible only in a narrow range of photon-energies around $20 \mathrm{eV}$, which corresponds to the $\Gamma$ point, and completely vanishes as the photon energy is changed. This indicates that on going along the $\Gamma-Z$ direction the dispersion crosses the chemical potential and that the FS is closed. On the other hand, for the high- $n$ samples, the bulk band remains visible for all photon energies. The maximal width of the bulk band is obtained at about $20 \mathrm{eV}$ ( $\Gamma$ point); the band then disperses upward towards a minimum at both zone boundaries located at photon energies of about $14.5 \mathrm{eV}$ and $23.5 \mathrm{eV}$. This is a clear indication of an open FS at high $n$.

Next, we look in more detail at the band structure of a $n \simeq 10^{20} \mathrm{~cm}^{-3}$ sample. Around the $\Gamma$ point the parabolic bulk 
(a)

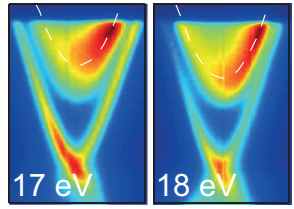

(b)

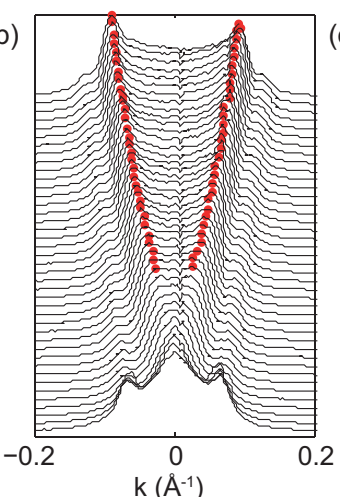

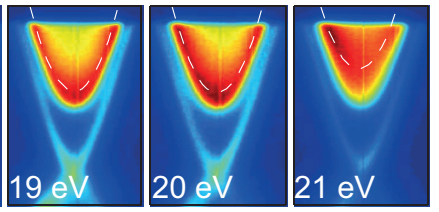

$\mathrm{k}_{\mathrm{z}}\left(\AA^{-1}\right)$

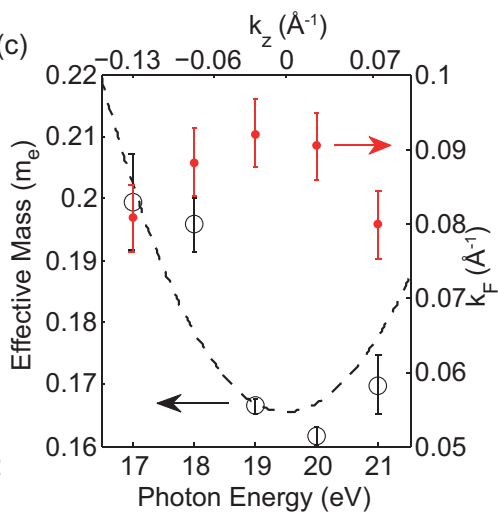

FIG. 5. (Color online) Effective mass of the bulk band. (a) Dispersion of the bulk band around the $\Gamma$ point, where the dispersion of the bulk is clear and allows an accurate measurement of the effective mass. The dashed line represents the parabolic fits to the dispersion. (b) MDCs for the 19-eV photon-energy data. The red points are the maxima of the MDCs. These maxima are used to extract the dispersion. (c) Summary of the fit results. The red points (black circles) represent $k_{F}$ (effective mass) as a function of the photon energy. (d) A close view of the FS calculated in Fig. 3(c). The color code corresponds to the effective mass calculated for the whole momentum range using the fit in Fig. 5(c). The cylindrical-type shape has a corrugation ratio of $\simeq 1.05$. This corrugation ratio together with the enhancement of the effective mass at the zone boundary explain the absence of a second frequency and the amplitude attenuation at high tilt angles as detailed in the text.

band is very clear; this can be seen in Figs. 5(a) and 5(b). By following the peaks in the momentum distribution curves (MDCs), we extract the band dispersion [Fig. 5(b)]. Fitting the data to a simple parabolic dispersion model, we can find $k_{F}$ and the effective mass at different photon energies. The parabolic best fits are shown as dashed lines in Fig. 5(a). The effective masses resulting from these fits are shown in Fig. 5(c) together with $\mathrm{k}_{F}$. We find that when moving away from the $\Gamma$ point towards the zone-boundary $k_{F}$ decreases and the effective mass increases substantially.

In Fig. 5(d) we show the same FS shown in Fig. 3(c) with a color code representing the effective mass, which is measured by ARPES [using the parabolic fit in Fig. 5(c)] for various $k_{z}$. Naively, for this type of FS one would expect two frequencies: the first from the maximal cross section, at the plane going through the $\Gamma$ point, and the second from the minimal cross section at zone boundary (the $Z$ points). The $k_{z}$ dependence of the effective mass extracted from the ARPES data suggests that the $\mathrm{SdH}$ signal arising from the minimal cross section at the zone boundary will be very weak, as the SdH intensity depends exponentially on the effective mass. This, together with our finding that the area of the cross section perpendicular to $k_{z}$ changes by merely $5 \%$, explains why the second frequency is absent in our measurements. Furthermore, as the angle $\theta$ with respect to the $\mathrm{C} 3$ axis increases, the $\mathrm{SdH}$ is probing parts of the FS at which the electron's effective mass is larger, so we expect the signal to become weaker, as observed.

The ARPES data suggests a slightly larger corrugation ratio compared to the $\mathrm{SdH}$ results, but overall the agreement between the ARPES and the SdH results is impressive. The two probes yield similar values of $k_{F}$ for the various samples (see Appendix D for more information), and both techniques show clearly a transition from a closed FS at low carrier concentrations to an open FS at high carrier density. In particular, all superconducting samples have an open FS.

\section{CONCLUSION}

In order for a time-reversal-invariant superconductor with odd-parity pairing to be a 3D topological superconductor, it must have a Fermi surface that encloses an odd number of time-reversal-invariant (TRI) momenta in the Brillouin zone. ${ }^{5}$ We show here that the FS of $\mathrm{Cu}_{x} \mathrm{Bi}_{2} \mathrm{Se}_{3}$ encloses two TRI points, $\Gamma$ and $Z$. Our results cast doubt on $\mathrm{Cu}$-doped $\mathrm{Bi}_{2} \mathrm{Se}_{3}$ as a possible realization of a TRI 3D topological superconductor. Interestingly, this material can be a realization of a 2D-like weak topological SC. Such a system is predicted to have counterpropagating edge states that can produce Andreev bound states but not on the (001) surface. If this is the case, the observed ZBCPs in recent point contact experiments ${ }^{3,4}$ can be a result of tunneling into crystalline facets exposing surfaces other than the (001) one.

\section{ACKNOWLEDGMENTS}

We are grateful to E. Berg for helpful discussions, S. Lerer for help with the numerical evaluation of the FS, D. Lonza for technical assistance at Elettra, and V. K. Guduru for help in the HFML. This work was supported by the Israeli Science Foundation. Work at Tel-Aviv University is supported by the Ministry of Science and Technology under Contract No. 3-8667. The Synchrotron Radiation Center is supported by NSF DMR 0084402. Part of this work was supported by the EuroMagNET II under the EU Contract No. 228043 and by EU Contract No. 312284. The work at Elettra has been supported by the European Community - FP7 CALIPSO (Contract No. 312284) Transnational Access Program. E.L. and E.M. contributed equally to this work. 


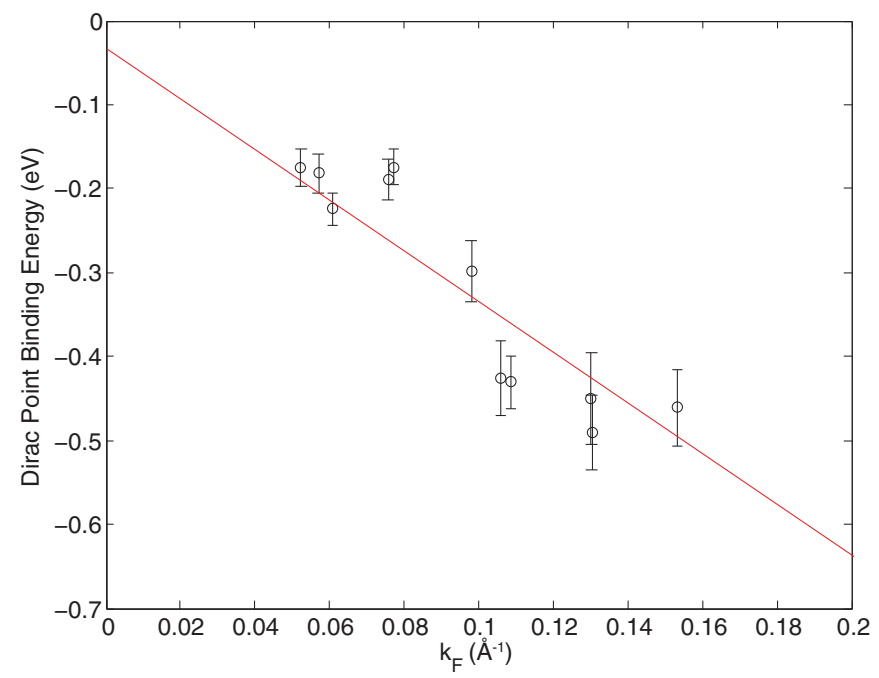

FIG. 6. (Color online) The energy position of the Dirac point and the value of $k_{F}$ for the different samples appearing in Fig. 1(d) of the main text. Error bars are 95\% confidence levels.

\section{APPENDIX A: RIGID SHIFT}

The data presented in Fig. 1(d) of the main text are cuts of the surface state in the $\Gamma K$ direction passing through the $\Gamma$ point. The dispersion was obtained by tracking the peak in the MDCs in the ARPES spectra. The energy at which we find the Dirac point and the Fermi momentum (of the surface state) for each of these samples is plotted in Fig. 6. There is a linear relation between these two quantities, which is expected in the case of a rigid shift of the chemical potential in a linearly dispersing band, where the chemical potential is determined by the carrier density. The scatter in the plot is a result of slight misalignment of the different samples.

\section{APPENDIX B: INNER POTENTIAL}

We relate the value of $k_{z}$ for electrons inside the crystals to their kinetic energy after photoemission using the freeelectron final-state approximation $k_{z}=\sqrt{2 m / \hbar^{2}\left(E_{\mathrm{kin}}+V_{0}\right)}$. The mapping of band dispersion in the $k_{z}$ direction is then carried out by changing the photon energy used in the photoemission process, thus changing the value of $E_{\mathrm{kin}}$ and obtaining a different value for $k_{z}$. The constant $V_{0}$ is specific to the material and is called the inner potential; formally it is given by $V_{0}=\mu+\phi$, where $\mu$ is the chemical potential measured from the bottom of the band and $\phi$ is the work function. We can determine the value of $V_{0}$ experimentally from the ARPES data, similarly to what appears in Fig. 4 of the main text, by identifying the high-symmetry points in the dispersion along $k_{z}$. In Fig. 7 we plot the position of the high-symmetry points as a function of photon energy and obtain a value of approximately $V_{0} \simeq 10.3 \mathrm{eV}$.

\section{APPENDIX C: BAND DISPERSION ALONG $k_{z}$}

To make a more quantitative analysis, we need to find the bottom of the band for each $k_{z}$ cut. This is found to be a tricky task, and for some photon energies, a clear parabolic dispersion is seen in the data but for other photon energies we

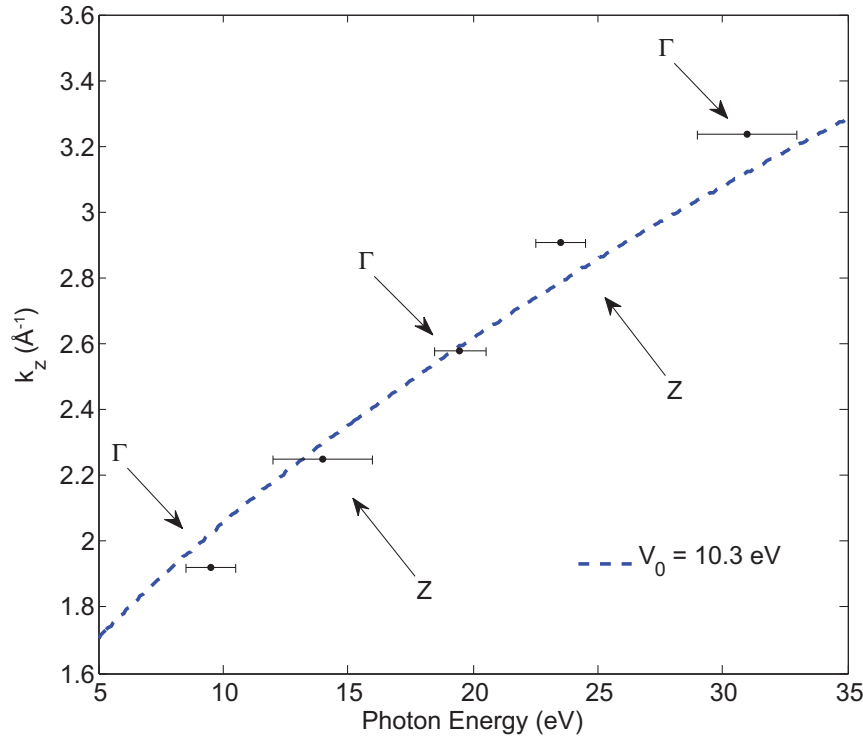

FIG. 7. (Color online) Finding the inner potential $V_{0}$ : The high symmetry points are identified from the photon energy scans, similarly to what is shown in Fig. 4 of the main text. The $k_{z}$ axis separation between the data points is taken to be the $\Gamma-Z$ distance, and the error bars show the uncertainty in the position. The dashed line is the equation of free-electron final-state approximation (see text) plotted with $V_{0}=10.3 \mathrm{eV}$.

find a parabolic region "filled" with almost constant intensity. We look for the energy for which the intensity drops to half its maximal value; this criterion is used for the entire data set. The $k_{z}$ values were calculated using the free-electron final-states assumption with an inner potential $V_{0}=10.3 \mathrm{eV}$.

In Fig. 8, we plot the dispersion of the bulk band along $k_{z}$, for a few samples with different chemical potential values. The data for the different samples are plotted relative to the bottom of the band for each sample. The horizontal solid lines represent the chemical potential for each sample measured

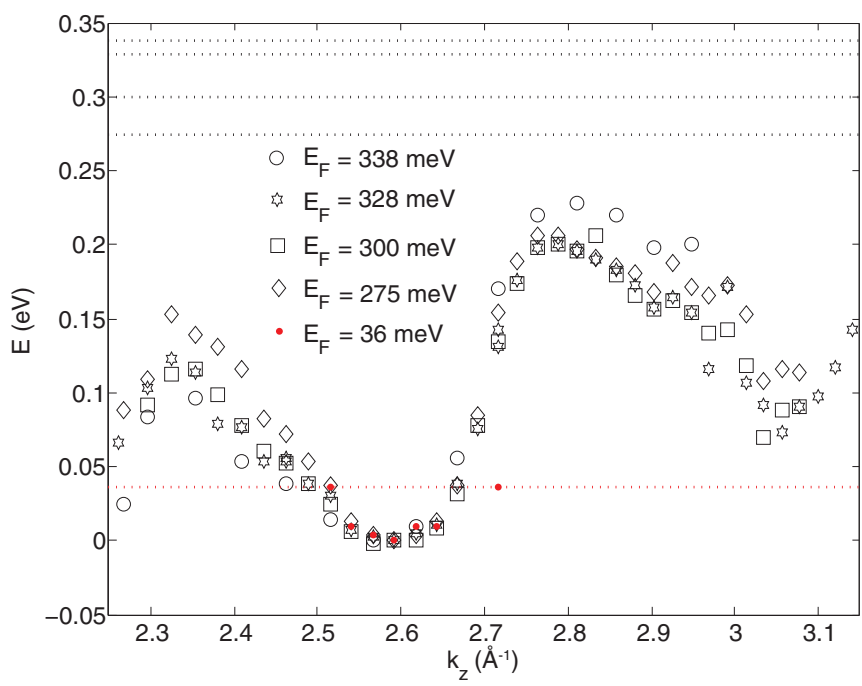

FIG. 8. (Color online) Dispersion of the bulk band along the $k_{z}$ axis. The plot contains data from five different samples with varying Fermi energies (noted by the horizontal dash lines) as measured from the bottom of the bulk band at the $\Gamma$ point. 
relative to the bottom of the band. One can see that the band shifts rigidly with the increasing doping level; this is apparent in the way the data for different samples coincide. The dispersion of the different samples is identical going from the $\Gamma$ point toward the upper $Z$ point. Going from the $\Gamma$ point down, the agreement is not as good. This might be a result of the way we define the bottom of the band. In addition, the free-electron final-state approximation can lead to errors.

The dispersion of the sample with the low chemical potential (36 meV) crosses the Fermi level, an indication of a closed FS. For the other samples, we do not find a crossing of the Fermi level, an indication of an open FS. Furthermore, for the highly doped samples we find a saturation of the occupied band width. This is again in agreement with an open FS and the absence of a Fermi crossing point along $\Gamma-Z$.

\section{APPENDIX D: LOWER LIMIT FOR $\boldsymbol{k}_{F}^{z}$}

We make a simple estimation of $k_{F}$ in the $z$ direction, which does not require a measurement of the dispersion along $k_{z}$. The surface-state FS always encloses the bulk FS, even for highly doped samples. The $k_{F}$ we find for the surface state does not depend on the photon energy. We can use this value as an upper limit for the $k_{F}$ of the bulk band in the $k_{x} k_{y}$ plane. Knowing the carrier density (from Hall measurements), one can calculate the Luttinger FS volume. Assuming an ellipsoidal FS, $\frac{3 \pi^{2} n}{k_{F}^{2}}$ gives a lower limit for $k_{F}$ in the $k_{z}$ direction.

In Fig. 9, we show the carrier density extracted from the Hall measurements as a function of the surface state $k_{F}$ for various samples. The solid line is the carrier density expected for a spherical Fermi surface with a radius corresponding to $k_{F}$.

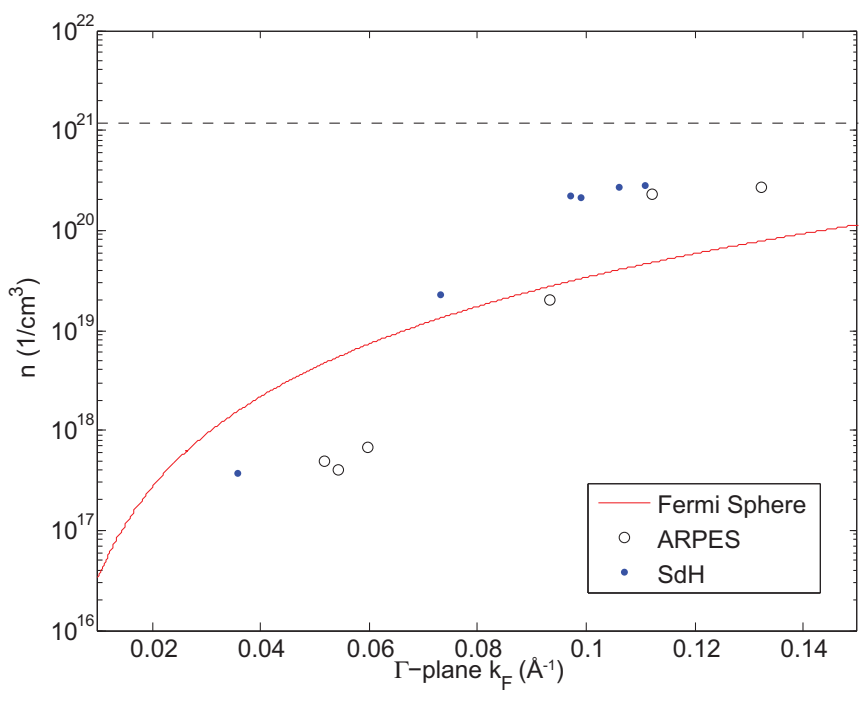

FIG. 9. (Color online) Carrier density as a function of $k_{F}$. The carrier densities are obtained from Hall measurements and they span nearly three orders of magnitude. The $k_{F}$ (blue dots) was obtained from $\mathrm{SdH}$ data, measured with magnetic field parallel to the $\mathrm{C} 3$ axis. The $k_{F}$ (hollow black circles) is that of the surface state obtained from the ARPES data, and it represents an upper bound on the $k_{F}$ of the bulk. The solid line is the expected carrier density for a spherical Fermi surface. The dashed line is the carrier density for which the radius of the Fermi sphere equals the $\Gamma-Z$ distance, that is, the Fermi surface reaches the edge of the first Brillouin zone.
The data points for samples with low carrier density lie slightly below the line, as expected, whereas the points representing high density samples are found above the line. Furthermore, the values we get for $k_{F}$ in the $k_{z}$ direction for the samples with $n \sim$ $10^{20} \mathrm{~cm}^{-3}$ are larger than the $\Gamma-Z$ distance, indicating that the FS crosses the top zone boundary, meaning that the FS is open.

The blue dots in Fig. 9 are the $k_{F}$ from the $\mathrm{SdH}$ data, one can clearly see the good agreement between the values obtained using ARPES and $\mathrm{SdH}$.

\section{APPENDIX E: CORRUGATED CYLINDER MODEL}

We use a simple tight-binding model to fit the $\mathrm{SdH}$ data. Expanding around $k_{x} \simeq k_{y} \simeq 0$, we get the equation describing the entire Fermi surface:

$$
\frac{1}{2} t_{\perp}\left(k_{x}^{2}+k_{y}^{2}\right) a^{2}+t_{z}\left[1-\cos \left(k_{z} c\right)\right]=\varepsilon_{F},
$$

where $a$ and $c$ denote the crystals constants in the $x y$ plane and $z$ axis, respectively, and $t_{\perp}$ and $t_{z}$ denote the transfer integrals in the $x y$ plane and $z$ axis, respectively, where $t_{\perp}>t_{z}>0$.

Applying a magnetic field directing at an angle $\theta$ relative to the $z$ axis and due to symmetry in the $x y$ plane (taking $\phi=0$, where $\phi$ is the angle in the $x y$ plane), the equation for the Fermi surface boundary of the cross sectional region is

$$
\frac{1}{2} t_{\perp}\left(k_{x}^{2}+k_{y}^{2}\right) a^{2}+t_{z}\left[1-\cos \left(k_{x} c \tan \theta\right)\right]=\varepsilon_{F} .
$$

Measuring wave numbers in units of $a^{-1}$ and energies in units of $t_{\perp}$ this equation becomes

$$
\begin{aligned}
& k_{x}^{2}+k_{y}^{2}+2 \eta\left[1-\cos \left(k_{x} \gamma \tan \theta\right)\right]=2 \varepsilon_{F}, \\
& \eta \equiv \frac{t_{z}}{t_{\perp}}<1, \gamma \equiv \frac{c}{a}>1,
\end{aligned}
$$

The solutions for the cross sectional curve is

$$
k_{y}= \pm \sqrt{2 \varepsilon_{F}-k_{x}^{2}-2 \eta\left[1-\cos \left(k_{x} \gamma \tan \theta\right)\right]} .
$$

Finally, we calculate the cross-sectional area perpendicular to the magnetic field. Using the Onsager relation, the corresponding frequency is given by

$$
\begin{aligned}
F(\theta, \eta)= & \frac{\hbar}{2 \pi e a^{2}} \int k_{y} d\left(k_{x} / \cos \theta\right) \\
= & \frac{1}{\cos \theta} \frac{\hbar}{2 \pi e a^{2}} \\
& \times \int \sqrt{2\left(\varepsilon_{F}-\eta\right)-k_{x}^{2}+2 \eta \cos \left(k_{x} \gamma \tan \theta\right)} d k_{x} .
\end{aligned}
$$

Expressing the Fermi energy using the zero angle frequency (where the magnetic field is perpendicular to the $x y$ plane),

$$
F_{0} \equiv F(\theta=0, \eta)=\frac{\hbar \varepsilon_{F}}{e a^{2}} .
$$

The final equation for the angular dependence of the $\mathrm{SdH}$ frequency is

$$
\begin{aligned}
F(\theta, \eta)= & \frac{\Delta(\theta, \eta)}{\cos \theta} \\
\Delta(\theta, \eta)= & \frac{\hbar}{2 \pi e a^{2}} \int\left\{2\left(F_{0} e a^{2} / \hbar-\eta\right)\right. \\
& \left.-k_{x}^{2}+2 \eta \cos \left(k_{x} \gamma \tan \theta\right)\right\}^{1 / 2} d k_{x} .
\end{aligned}
$$



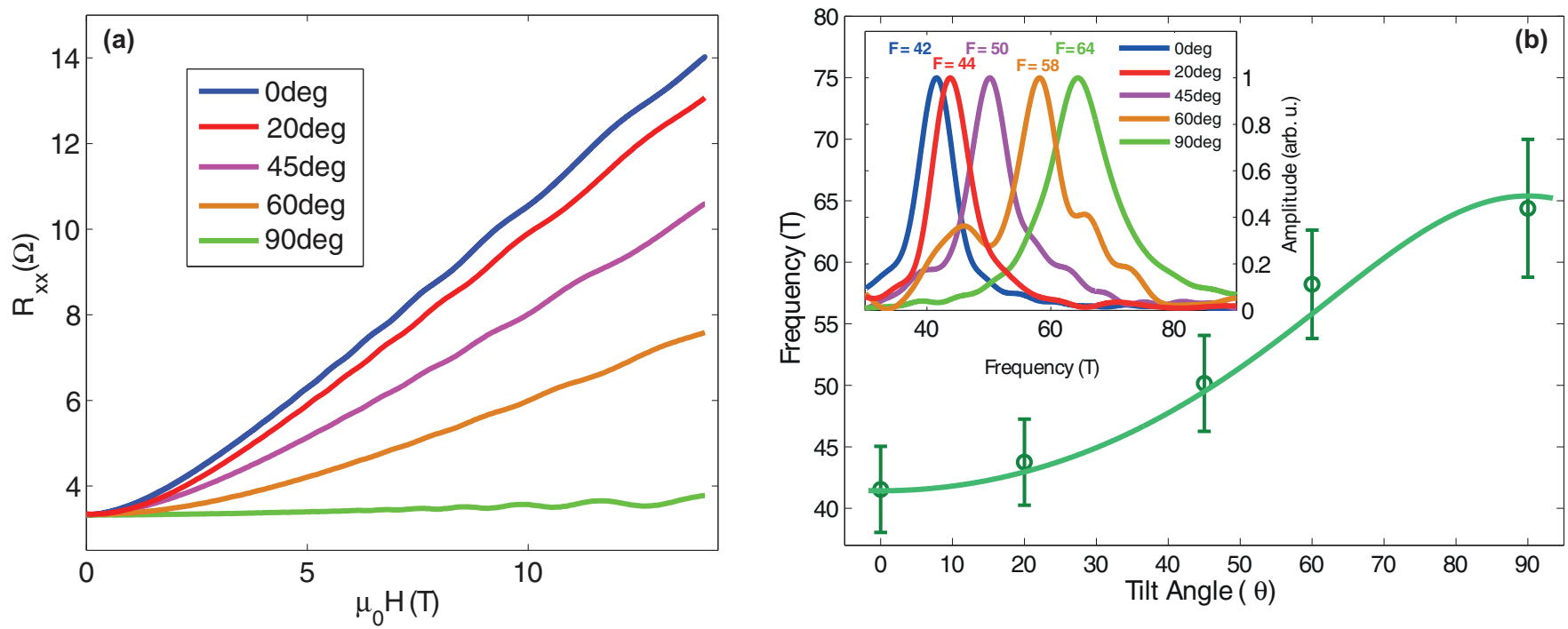

FIG. 10. (Color online) Sample with $n 3 D \simeq 10^{17}$. (a) Longitudinal resistance versus magnetic field at various tilt angles between the C3 axis and the magnetic field. (b) Frequency versus tilt angle. The solid line is a fit for an ellipsoidal Fermi surface model. Inset: FFT analysis of each tilt angle.

Performing a numerical integration (on $k_{x}$ ) combined with a numerical fit (frequency versus magnetic field tilt angle), one can extract the desired parameter $\eta$ (which defines the corrugation of the cylinder). Our experimental data (the frequency $F$ at different magnetic field tilt angles $\theta$ ) enables us to perform this analysis. We note that this simple theoretical model does not allow us to find the dependence of the cyclotron mass on $k_{z}$ as experimentally determined in Fig. 5 in the paper.

\section{APPENDIX F: ADDITIONAL SDH DATA AND ANALYSIS}

In Figures 10, 11, and 12 we present the $\mathrm{SdH}$ measurement of three different samples with various carrier concentrations (presented in the paper itself). The measurements are at various tilt angles between the $\mathrm{C} 3$ axis and the magnetic field. For these measurements we present FFT after background substraction for each tilt angle and a fit for each samples Fermi surface.
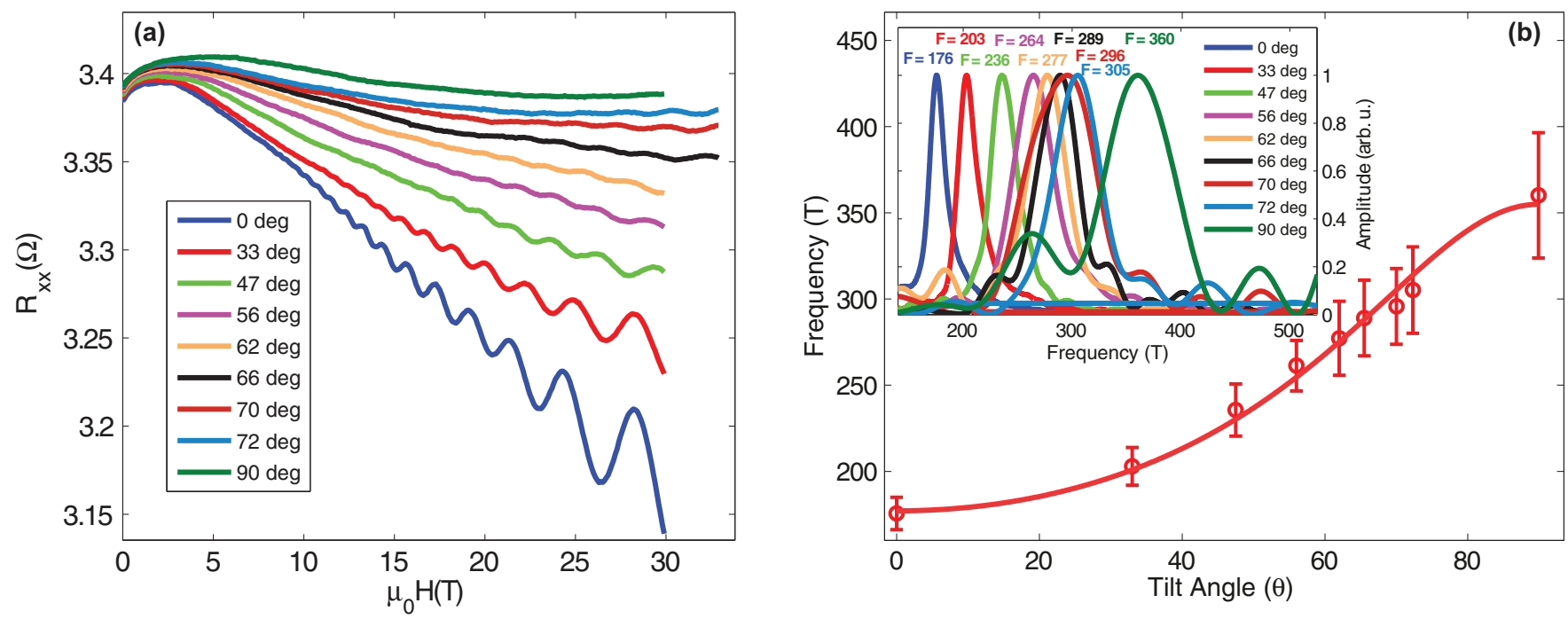

FIG. 11. (Color online) Sample with $n 3 D \simeq 10^{19}$. (a) Longitudinal resistance versus magnetic field at various tilt angles. $0^{\circ}$ corresponds to magnetic field parallel to the $\mathrm{C} 3$ axis. (b) Frequency versus tilt angle. The solid line is a fit for an ellipsoidal Fermi surface model. Inset: FFT analysis of each tilt angle. 

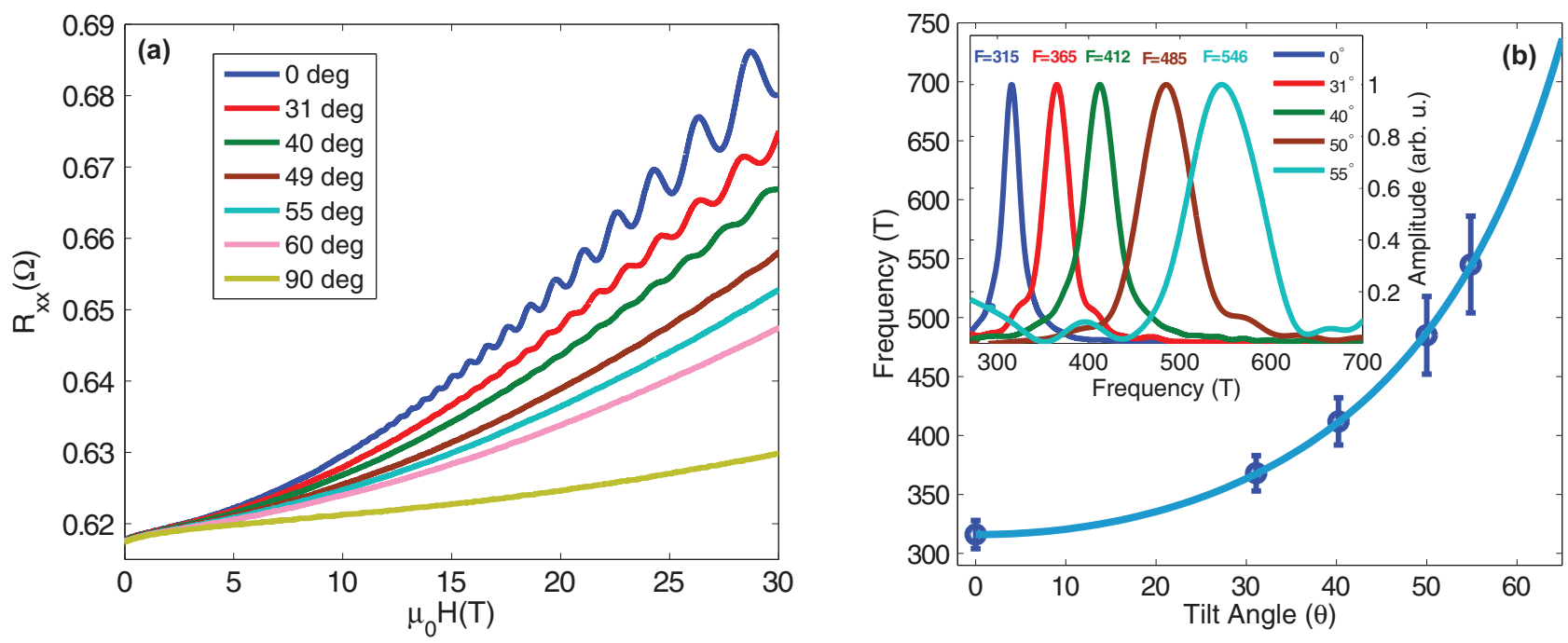

FIG. 12. (Color online) Sample with $n 3 D \simeq 10^{20}$. (a) Longitudinal resistance versus magnetic field at various tilt angles. (b) Frequency versus tilt angle. The solid line is a fit for a cylindrical Fermi surface model $\left[F \propto \frac{1}{\cos (\theta)}\right]$. Inset: FFT analysis for each tilt angle.

*yodagan@post.tau.ac.il

${ }^{1}$ X.-L. Qi and S.-C. Zhang, Rev. Mod. Phys. 83, 1057 (2011).

${ }^{2}$ M. Z. Hasan and C. L. Kane, Rev. Mod. Phys. 82, 3045 (2010).

${ }^{3}$ S. Sasaki, M. Kriener, K. Segawa, K. Yada, Y. Tanaka, M. Sato, and Y. Ando, Phys. Rev. Lett. 107, 217001 (2011).

${ }^{4}$ T. Kirzhner, E. Lahoud, K. B. Chaska, Z. Salman, and A. Kanigel, Phys. Rev. B 86, 064517 (2012).

${ }^{5}$ L. Fu and E. Berg, Phys. Rev. Lett. 105, 097001 (2010).

${ }^{6}$ T. H. Hsieh and L. Fu, Phys. Rev. Lett. 108, 107005 (2012).

${ }^{7}$ H. Zhang, C.-X. Liu, X.-L. Qi, X. Dai, Z. Fang, and S.-C. Zhang, Nat. Phys. 5, 438 (2009).

${ }^{8}$ Y. Xia, D. Qian, D. Hsieh, L. Wray, A. Pal, H. Lin, A. Bansil, D. Grauer, Y. S. Hor, R. J. Cava et al., Nat. Phys. 5, 398 (2009).

${ }^{9}$ Y. S. Hor, A. Richardella, P. Roushan, Y. Xia, J. G. Checkelsky, A. Yazdani, M. Z. Hasan, N. P. Ong, and R. J. Cava, Phys. Rev. B 79, 195208 (2009).

${ }^{10}$ Y. Hor, J. Checkelsky, D. Qu, N. Ong, and R. Cava, J. Phys. Chem. Solids 72, 572 (2011).

${ }^{11}$ L. A. Wray, S.-Y. Xu, Y. Xia, Y. S. Hor, D. Qian, A. V. Fedorov, H. Lin, A. Bansil, R. J. Cava, and M. Z. Hasan, Nat. Phys. 6, 855 (2010)
${ }^{12}$ H. Kohler, Phys. Status Solidi B 62, 57 (1974).

${ }^{13}$ P. Larson, V. A. Greanya, W. C. Tonjes, R. Liu, S. D. Mahanti, and C. G. Olson, Phys. Rev. B 65, 085108 (2002).

${ }^{14} \mathrm{D}$. Shoenberg, Magnetic Oscillations in Metals (Cambridge University Press, Cambridge, 1984).

${ }^{15}$ J. G. Analytis, J.-H. Chu, Y. Chen, F. Corredor, R. D. McDonald, Z. X. Shen, and I. R. Fisher, Phys. Rev. B 81, 205407 (2010).

${ }^{16}$ M. Bianchi, D. Guan, S. Bao, J. Mi, B. B. Iversen, P. D. King, and P. Hofmann, Nat. Commun. 1, 128 (2010).

${ }^{17}$ M. Petrushevsky, E. Lahoud, A. Ron, E. Maniv, I. Diamant, I. Neder, S. Wiedmann, V. K. Guduru, F. Chiappini, U. Zeitler et al., Phys. Rev. B 86, 045131 (2012).

${ }^{18}$ H. Kohler, Phys. Status Solidi B 58, 91 (1973).

${ }^{19}$ N. P. Butch, K. Kirshenbaum, P. Syers, A. B. Sushkov, G. S. Jenkins, H. D. Drew, and J. Paglione, Phys. Rev. B 81, 241301 (2010).

${ }^{20}$ K. Eto, Z. Ren, A. A. Taskin, K. Segawa, and Y. Ando, Phys. Rev. B 81, 195309 (2010).

${ }^{21}$ S. Hüfner, Photoelectron Spectroscopy (Springer, Berlin, 2003).

${ }^{22}$ This differs from the results in Ref. 17, where the oscillations followed $\frac{1}{\cos (\theta)}$ dependence up to $72^{\circ}$. 